\title{
Erektile Dysfunktion: Vorteile der intraurethralen Therapie nutzen
}

_ Bis zu $30 \%$ der europäischen Männer leiden an erektiler Dysfunktion (ED), sagte Dr. Axel-Jürg Potempa, München. Diese Erkrankung beeinträchtigt sexuelle und auch nicht sexuelle Lebensqualitätsaspekte erheblich. Die Rate der Patienten, die auf eine Behandlung mit Phosphodiesterase-5 (PDE-5)-Hemmer nicht ansprechen, bezifferte Potempa auf rund $25 \%$. Gründe für Therapieversagen seien unter anderem periphere Nervenschädigungen nach radikaler Prostatektomie, Diabetes mellitus sowie andere Erkrankungen mit Neuro- oder Vaskulopathien. Die Einnahme von Nitraten zählt zu den häufigsten Kontraindikationen gegen PDE-5-Inhibitoren. Mit Alprostadil (MUSE ${ }^{\oplus}$ ), das als Mikrozäpfchen mit einem kleinen Applikator in die Harnröhre eingebracht wird, steht eine leitliniengerechte lokale Behandlung zur Verfügung Die Daten mehrerer Studien zeigen, dass unter Alprostadil 75,4\% der Patienten einen Wert auf der Erection Assessment Scale von 3, 4 oder den Maximalwert 5 erreichten gegenüber 12,7\% unter Placebo. Mit 49,2\% erzielte knapp die Hälfte einen Wert von
4 oder 5 gegenüber $4,8 \%$ in der Placebogruppe [Costa P \& Potempa AJ. Drugs 2012; 72 : 2243-54]. Die günstige Wirkung von intraurethralem Alprostadil bei unterschiedlicher ED-Genese konnte bereits eine frühere Publikation belegen [Padma-Nathan $\mathrm{H}$ et al. New Engl J Med 1997; 336: 1-7].

Dr. Eckhard Kröger, Bad Wildungen, empfahl eine Anfangsdosierung von 500 Mg Alprostadil, später kann dann auf die wirksamere 1.000- $\mu \mathrm{g}$-Dosierung gewechselt werden. Häufig sei bereits nach nervschonender radikaler Prostatektomie eine Schwellkörperkonditionierung mit einem PDE-5-Hemmer für die Erektion nicht ausreichend; hier zeige Alprostadil eine gute additive Wirkung. Nach nicht nervschonender Operation empfahl er den primären Einsatz von Alprostadil.

Dr. Andreas Fischer

Presssekonferenz ${ }_{\text {,MUSE }}{ }^{\circledR}$ - Rennaissance eines bewährten Therapiekonzeptes zur Behandlung der ED", München,

19. März 2013,

Veranstalter: MEDA Pharma, Bad Homburg
Pfizer und Ipsen kooperieren im Außendienst

Mit einer Kooperation in den urologischen Außendiensten haben Pfizer Pharma und Ipsen Pharma ihre Kompetenzen im deutschen Markt erweitert. So werden die Außendienste beider Unternehmen ab April für das Pfizer-Anticholinergikum Toviaz ${ }^{\circledR}$ (Fesoterodin) für die Behandlung der überaktiven Blase $(O A B)$ und das von Ipsen zur Behandlung von Prostatakarzinomen entwickelte Pamorelin ${ }^{\circledast}$ (Triptorelin) zuständig sein. Für niedergelassene Urologen bieten Ipsen und Pfizer damit eine umfassendere urologische Beratungskompetenz und größere Kundennähe.

Fesoterodin wird seit der Zulassung im Jahr 2007 bei Patienten mit überaktiver Blase eingesetzt, um erhöhte Harnfrequenz, Harndrang und Dranginkontinenz, symptomatisch zu behandeln. Bei Fesoterodin handelt es sich um einen Muskarinrezeptor-Antagonisten mit einem dosisproportionalen Verhalten. Somit ist es möglich, die Dosis individuell an die Bedürfnisse des jeweiligen Patienten anzupassen. Triptorelin ist ein LHRH-Analogon zur Behandlung des lokal fortgeschrittenen oder metastasierenden Prostatakarzinoms. Triptorelin ist als 1-, 3- und 6-Monatsdepot verfügbar. Es ist weltweit in über 60 Ländern zugelassen und gehört europaweit zu den führenden LHRH-Analoga.

Nach Informationen von

Pfizer, Berlin, und Ipsen, Ettlingen

Blasenbeschwerden gegensteuern

Jede fünfte Frau leidet unter Blasenschwäche, verspürt ständigen Harndrang oder ungewollten Harnabgang. Medizinische Präparate aus bewährten Heilpflanzen können dabei helfen, Problemen mit dem Wasserlassen wirksam und verträglich gegenzusteuern. GRANU FINK ${ }^{\circledR}$ femina wirkt mit seiner einzigartigen Wirkstoffkombination und kräftigt das Blasen-BeckenbodenSystem betroffener Frauen: Der FINK ${ }^{\circledR}$ Arzneikürbis stärkt die Blasenmuskulatur, Gewürzsumach (Rhus coriaria) wirkt reizlindernd und unterstützt so die Normalisierung des Harndrangs, Hopfen beruhigt die nervöse Blase. Wie eine wissenschaftliche Studie nachgewiesen hat, tritt bereits nach kurzer Zeit eine spürbare Besserung ein [Schilcher H \& Bauer HW. Ärztezeitschrift für Naturheilverfahren 2003; 44: 27-30].

Nach Informationen von

Deutsche Chefaro Pharma, Düsseldorf 